\title{
DNA Molecule as a Spin System and the Symmetric Top Model
}

\author{
Subhamoy Singha Roy \\ Department of Physics, JIS College of Engineering (Autonomous), West Bengal University of Technology, \\ Kalyani, Nadia -741235, India \\ Email: ssroy.science@gmail.com
}

\begin{abstract}
It is observed that when the conformational properties of DNA molecule are mapped onto an antiferromagnetic spin system we can realize the specific properties of the rod-like-chain (RLC) model where the analytically continued partition function corresponds to the symmetric top Hamiltonian. The relevant path integral represents a charge particle in the field of a non-quantized monopole which suggests that angular momentum is not quantized. However in a spin chain the nonquantized monopole charge appears in the renormalization group flow and corresponds to the Barry phase required by a spin $1 / 2$ state in an entangled spin system. Thus avoids the RLC model crisis.
\end{abstract}

Keywords: Spin system, symmetric top, monopole

PACS: $87.15 .-\mathrm{V}$

The topological and elastic properties of a DNA molecule have been studied earlier by several authors using quantum mechanics. Klolodenko and Vilgis [1] studied the elastic response to elongation force for polymer chains of arbitrary stiffness using the relation between semiflexible polymers and Euclidean Dirac fermions. In case of a DNA molecule the worm-like-chain (WLC) model was introduced [2] which describes a chain by an elastic continuous curve at thermal equilibrium with a single elastic constant, the persistent length A characterizing the bending energy. The WLC can be solved analytically by mapping it onto a quantum mechanical problem. Indeed the partition function is a Euclidean path integral for a quantum dumbbell. Bouchiat and Mezard [3] generalized this model introducing twist rigidity $C$ and it appears that a DNA molecule can be depicted as a thin elastic rod. This rod-likechain (RLC) model is characterized by the fact that the partition function can be mapped onto the path integral representing a charged particle in the field of a non-quantized monopole. When we describe it in terms of three Euler angles, the corresponding Hamiltonian is found to be singular and needs a small distance cut-off which is the natural length scale of the double helix pitch. The RLC model is able to reproduce the experimentally observed results relating the effects of supercoiling in the elongation force characteristics in the small supercoiling region. A given configuration of the RLC is specified in the continuum limit by the local orthonormal trihedron $\left\{\vec{e}_{i}(s)\right\}=\{\vec{u}(s), \vec{n}(s), \vec{t}(s)\}$ where $s$ is the arc length along the molecule. Here $\vec{u}(s)$ is the unit vector along the basis line and $\vec{t}(s)$ is the tangent to the chain and $\vec{n}(s)=\vec{t}(s) \Lambda \vec{u}(s)$. The evolution of the trihedron $\left\{\vec{e}_{i}(s)\right\}$ along the chain is obtained by applying the rotation $R(s)$ to a reference trihedron $\left\{\vec{e}_{i}^{0}(s)\right\}$ attached to a rectilinear relaxed molecule. The rotation $R(s)$ is parametrized by three Euler angles $\theta(s), \phi(s)$ and $\psi(s)$. The reference trihedron is characterized by $\theta(s)=0, \phi(s)+\psi(s)=\omega_{0} s$ where $\omega_{0}$ is the rotation per unit length of the base axis. For given values of the Euler angles $\theta(s), \phi(s)$ and $\psi(s)$ at the two ends $s_{0}=0$ and $s_{1}=L$ of the chain the partition function is given by

$$
\left(\theta_{1}, \phi_{1}, \psi_{1}, s_{1} \mid \theta_{0}, \phi_{0}, \psi_{0}, s_{0}\right)=\int D(\theta, \phi, \psi) \exp \left(-E_{R L C} / k_{B} T\right)
$$

The elastic energy $E_{R L C}$ is transformed into the integral $i \int_{t_{0}}^{t_{1}} d t L(t)$ where $L(t)$ is that of a symmetric top in a static electric field. The analytically continued partition function corresponds to the Feynman amplitude 


$$
\begin{aligned}
\left\langle\theta_{1}, \phi_{1}, \psi_{1}, s_{1} \mid \theta_{0}, \phi_{0}, \psi_{0}, s_{0}\right\rangle & =\int D(\theta, \phi, \psi) \exp \left(i \int_{t_{0}}^{t_{1}} d t L(t)\right) \\
& =\left\langle\theta_{1}, \phi_{1}, \psi_{1}\left|\exp -i\left(t_{1}-t_{0}\right) H_{t o p}\right| \theta_{0}, \phi_{0}, \psi_{0}\right\rangle
\end{aligned}
$$

where $H_{t o p}$ is the symmetric top Hamiltonian operator acting on Euler angles wave function. However it should be observed that the analogy is a bit formal. In fact in case of symmetric top the space is that of $2 \pi$ periodic functions of the Euler angles $\phi$ and $\psi$ but in the RLC model the space is that of general functions without any constraint of periodicity. It should be noted that the elastic theory cannot be valid in the atomic scale. In fact the corresponding Hamiltonian is singular and needs a small distance cut-off which is of the order of double helix pitch. One may also argue that the RLC model ignores the helical structure of the DNA molecule.

Recently a theory of the denaturation transition of a DNA molecule has been proposed in the framework of the mapping of the conformational properties of DNA onto a Heisenberg spin system[4]. As two polynucleotide chains are twisted about the molecule axis with a specific helical sense in a DNA molecule, this can be viewed as if a spin with a specific orientation is inserted on the axis such that two adjacent coils have opposite orientations of the spin. In fact with each turn two strands move in the opposite side of the axis and so the spin orientation assigned for the two adjacent coils should be opposite to each other. Indeed twisting of the two strands in mutually opposite directions can be taken to imply that two strands can be designated by two spins having orientations $+1 / 2$ and $-1 / 2$. When these two spins having opposite orientations are inserted on the axis such that these are located in the two adjacent sites with lattice spacing of one period of helix this represents an antiferromagnetic spin system. In this scenario denaturation transition can be formulated in terms of quantum phase transition induced by a quench when the temperature effect is incorporated in the quench time and torsion takes the role of the external field. In a recent letter [5] we have computed the melting profiles for the different sequence specific DNA molecules and the results are found to be in excellent agreement with experiment.

In the depiction of DNA molecule as an antiferromagnetic spin chain we observe that the spin degrees of freedom appear to be associated with three Euler angles $\theta, \phi$ and $\psi$ corresponding to an extended body. In fact it has been shown in earlier papers [6,7] that the quantization of a fermion in the framework of Nelson's stochastic quantization procedure [8] can be achieved when we introduce an internal variable which appears to be a direction vector depicting the spin degrees of freedom. The direction vector effectively corresponds to a vortex line which is topologically equivalent to a magnetic flux line. When we introduce the direction vector attached to a space-time point the spherical harmonics associated with the system incorporate apart from the polar angles $\theta$ and $\phi$, and another angle $\psi$ which is related to the rotational orientation of the direction vector. These three angles correspond to three Euler angles associated with an extended body. Thus the spin degrees of freedom introduce three Euler angles in the system. Indeed as a spin degree of freedom appears as $S U(2)$ gauge bundle and the group $S U(2)$ is topologically equivalent to the 3 -sphere $S^{3}$, we note that $S^{3}$ incorporates an extended body having three Euler angles $\theta, \phi, \psi$. It is observed that the spherical harmonics incorporating these three Euler angles correspond to monopole harmonics as the eigenvalue of the operator i $i \hat{\partial} / \partial \psi$ corresponds to a monopole charge. Indeed we can write the corresponding harmonics as $Y_{l}^{m, n}$, $m(\mu)$ being the eigenvalue of the operator $\left.i \hat{\partial} / \partial \phi^{(i} \hat{\partial} / \partial \psi\right), l$ being the angular momentum [9].

This suggests that the elasticity parameters depicted in terms of these angles have a correspondence with those derived in terms of a spin chain. So we can map the elasticity parameters derived from the spin chain on those derived in the RLC model. However we note that unlike the RLC model, the helical structure of DNA is the basic ingredient of the spin chain model. Also the lattice constant which is of the order of a helix period appears to be a natural cut-off which is needed to study the elastic properties in terms of the symmetric top Hamiltonian as is envisaged in the RLC model.

It should be mentioned that the analogy of the RLC model with the quantum symmetric top is confronted with a "crisis" in the sense that the RLC analog of the angular momentum is not quantized 
as the physical states of an elastic rod representing a double helix are not invariant under $2 \pi$ rotation about its axis. This phenomenon is known as the "RLC model crisis". In this context we may add that the Dirac quantization condition suggests that the monopole charge $\mu$ takes the values $0, \pm 1 / 2, \pm 1, \pm 3 / 2 \ldots \ldots$. However in an entangled spin system the monopole charge is found to be related to the measure of entanglement of a pair of nearest neighbour spins given by concurrence and undergoes a renormalization group $(\mathrm{RG})$ flow $[10,11]$.

The rules for the RG flow of the monopole charge known as the $\mu$-theorem are as follows. When the monopole charge depends on a certain parameter $\lambda$ we have

1) $\mu$ is stationary at fixed points $\lambda^{*}$ of the RG flow

2 ) at the fixed points $\mu\left(\lambda^{*}\right)$ is equal to the monopole charge given by quantized values $(\mu=0, \pm 1 / 2, \pm 1 \ldots \ldots)$

3) $\mu$ decreases along the RG flow i.e. $L(\partial \mu / \partial L) \leq 0$ where $L$ is a scale parameter.

In view of this analysis we note that the non-quantized angular momentum related to the violation of rotational invariance in RLC model can be associated with the RG flow of the monopole charge when it is mapped onto an antiferromagnetic spin chain. Indeed Bouchiat and Mezard [3] have pointed out that the writhe partition function Fourier transform corresponds to the quantum mechanical problem of a charged particle in the field of a monopole with non-quantized charge. These authors have prescribed a local writhe formula in terms of the Euler angles $\phi(s)$ and $\psi(s)$ defining a line integral

$$
\chi=\phi(L)+\psi(L)=\int_{0}^{L} d s(\dot{\psi}+\dot{\phi})
$$

The total twist can be written as

$$
T w=\int_{0}^{L} d s(\dot{\psi}+\dot{\phi} \cos \theta)
$$

A "local writhe" $\chi W$ is defined as

$$
\chi W=\chi-T w=\int_{0}^{L} d s \dot{\phi}(1-\cos \theta)
$$

They have shown that $\chi W$ can be written as the line integral

$$
\int d \phi A_{\varphi}=\int \vec{A}_{m}(\vec{r}) d \vec{r}
$$

where $A_{\phi}=(1-\cos \theta)$ is identified as the $\phi$-component of the potential vector $\vec{A}_{m}(\vec{r})$ of a magnetic monopole of charge unity. In fact for a closed circuit we have the relation

$$
\int_{0}^{2 \pi} A_{\phi} d \phi=2 \pi(1-\cos \theta)
$$

It is observed that the holonomy given by eqn.(7) effectively corresponds to the Berry phase [12] acquired by a quantum state while traversing a closed path involving a monopole of charge unity. In fact the Berry phase acquired by a spin state in a spin $1 / 2$ chain when the Hamiltonian is parallel transported along a closed circuit is given by $\mu \Omega(c)$ where $\Omega(c)$ is the solid angle subtended by the contour at the center of a unit sphere with a monopole of charge $\mu$ at the center. Noting that

$$
\Omega(c)=\int(1-\cos \theta) d \phi=2 \pi(1-\cos \theta)
$$

and for the monopole charge $\mu=1 / 2$, we have the Berry phase attained by a spin $1 / 2$ state in a chain while traversing a closed path given by [13.14]

$$
\phi_{B}=\pi(1-\cos \theta)
$$

It is noted that the holonomy given by eqn.(7) is twice this phase factor. It is observed that when a quantum state encircles a closed loop enclosing magnetic flux lines associated with the monopole of charge $\mu$ it acquires the Berry phase apart from dynamical phase when the phase is given by $\phi_{B}=2 \pi \mu$ [15]. Equating this with the expression given by eqn. (9) we note that the effective monopole charge associated with a spin state in an entangled spin system is given by the relation 


$$
\mu_{\text {eff }}=(1 / 2)(1-\cos \theta)
$$

Evidently the monopole charge takes non-quantized value apart from the situation when the polar angle $\theta$ of the spin axis with the quantization axis is given by $\theta=0, \pi / 2$ and $\pi$. This nonquantized monopole charge takes values on the $R G$ flow of the monopole charge in an entangled spin system [11]. Thus we observe that when we map a DNA molecule onto an antiferromagnetic spin system the apparent RLC model crisis appears to be associated with the $R G$ flow of the monopole charge in an entangled spin system. In view of this we note that the specific properties of the symmetric top model can be mapped onto an antiferromagnetic spin system without any ambiguity.

In summary it is here observed that when the conformational properties of a DNA molecule are mapped onto an antiferromagnetic spin chain we can realize the salient features of the RLC model when the analytically continued partition function corresponds to the symmetric top Hamiltonian acting an Euler angle wave function. The relevant path integral represents a charged particle in the field of a nonquantized monopole which suggests that the angular momentum is not quantized. However when a DNA molecule is mapped onto an antiferromagnetic spin chain the non-quantized monopole charge is found to be associated with the $R G$ flow of the monopole charge in an entangled spin system and thus avoids the RLC model crisis.

\section{References}

1. K.A.Kholodenko and T.Vilgis: Phys.Rev.E 50, 1257(1994).

2. M.Fixman and J.Kovac: J.Chem. Phys. 58, 1564 (1973).

3. C.Bouchiat and M.Mezard:Phys.Rev.Lett. 80, 1556(1997); Euro.Phys.J.E, 2, 377(2000).

4. S.S. Roy and P.Bandyopadhyay, Phys. Lett. A 337, 2884 (2013).

5. S.S. Roy and P.Bandyopadhyay, Europhys.Lett.109, 48002 (2015).

6. P.Bandyopadhyay and K.Hajra: J.Math.Phys. 28, 711 (1987).

7. K.Hajra and P.Bandyopadhyay: Phys.Lett. A 155, 7 (1991).

8. E.Nelson: Phys.Rev.150, 1079(1966); Dynamical theory of Brownian motion (Princeton University Press, Princeton, N.J.(1967).

9. C.A. Hurst: Ann.Phys 50, 37 (1968).

10. P.Bandyopadhyay: Int.J.Mod.Phys.A 15, 1415 (2000).

11. P.Bandyopadhyay: Proc.Roy.Soc (London) A 467, 427(2011).

12. M.V.Berry, Proc.Roy.Soc(Londan)A, 392, 45(1984).

13. B.Basu and P.Bandyopadhyay, Int. J.Geo. Meth. Mod. Phys. 9707 (2007).

14. B.Basu and P.Bandyopadhyay, J.Phys. A. 41, 055301 (2008).

15. D.Banerjee and P.Bandyopadhyay: J.Math.Phys.33, 990 (2012). 